\title{
Annual Scientific Meeting, Winnipeg, September 22-25, 2005
}

\section{Natural History of Peanut Allergy}

R. Borici-Mazi, J.A. Mazza, D.W. Moote, K. Payton, Division of Allergy and Clinical Immunology, University of Western Ontario, London, ON

Background: Peanut allergy affects 0.5 to $1 \%$ of the general population. Despite enormous efforts to educate peanut allergic patients and their families and increasing public awareness of hidden sources of peanuts, accidental exposures to peanuts still happen. The aim of this study was to calculate the cumulative risk of accidental exposures to peanut among peanut allergic patients and to study their effect on peanut-specific IgE levels (PNIgE). Methods: This was a retrospective chart review of peanut allergic patients followed up at LHSC from 1997 to 2004. Peanut allergic patients who were less than 15 years old at the time of first reaction to peanut and had serial measurements of PN-IgE were included in the study. Diagnosis of peanut allergy was based on significant history of acute reaction and positive SPT/PN-IgE to peanut. Statistical Analysis: Cumulative risk of accidental exposures and time to significant change of PNIgE were calculated using Kaplan-Meiers survival curves. Comparisons were made using the log-rank statistics. Results: Medical charts from 118 peanut allergic patients were reviewed (median age at first reaction was 1.5 years old, baseline PN-IgE value was $18.75 \mathrm{kU}_{\mathrm{A}} / \mathrm{L}$ ). Ninety-four patients decreased their PN-IgE levels, whereas 24 experienced elevation of PN-IgE. There were 88 reported accidental exposures in 64 patients and 17 patients experienced more than one accidental exposure. Calculated cumulative risk of reported accidental exposures at 1,5 and 10 years post initial reaction was $24.8 \%, 34 \%$ and $65.1 \%$, respectively. Conclusion: The predicting factors that determine resolution vs persistence of peanut allergy remain unknown and further studies are required. In conjunction with the avoidance of peanuts, education concerning early recognition of accidental exposures and knowledge of how to manage such reactions remain the main focus of peanut allergic patients' care.

\section{Improving the Quality of Life and Decreasing Pediatric Asthma Severity after an Emergency Department (ED) Educational Program}

R.B. Boychuk, Professor of Pediatrics and Surgery, University of Hawaii, John A Burns School of Medicine, Department of Pediatrics, Honolulu, HI. C.J. DeMesa, MPH, Program Coordinator, Kapiolani Medical Center for Women and Children, Emergency Department, Pediatric Asthma Program (HICARES), Honolulu, HI

The purpose of this program was to unite ED personnel, community physicians, and patients to integrate care by developing an effective educational asthma management program originating in the ED and extending to the primary care provider and family at four separate EDs on Oahu, Hawaii. Methods: This was a prospective cohort study of wheezing children 12 months to 18 years of age presenting to an ED. During Phase I (10/ $08 / 02$ to $10 / 01 / 03)$, baseline information collected included: signs and symptoms, peak flow use, type and frequency of medication use, use of a written asthma action plan (WAAP), past asthma history, NAEEP severity classification, and ED and discharge medications. Phase II (10/01/03 to 07/08/04) added a comprehensive, coordinated, standardized, ED educational interventional program for patients and families. Primary outcome measures included controller medication (CM) use, WAAP use, chronic severity classification, and validated QOL score. Results: In 313 intervention patients, daily CM use increased from $18.2 \%$ at ED encounter to $43.0 \%$ and $36.6 \% 3$ weeks and 3 months, respectively, postencounter. Analyzing the persistent categories 3 months post-intervention, the severe persistent category decreased from $73(23.3 \%)$ to $32(10.2 \%)$, while the moderate persistent category decreased from $47(15 \%)$ to 31 (9.9\%). Analysis of QOL scores revealed patients had significantly less daytime and nighttime coughing/ wheezing, and improved life activities (effect sizes: 0.83, $0.68,0.77$, respectively), including family life (0.74). Conclusion: This study provided an educational program that is safe, efficacious, and cost effective in changing 
the approach and delivery of medical care to patients with chronic asthma treated in the ED. This resulted in behavioral changes of physicians, caregivers parents, and asthmatic children, and improved CM adherence, clinical status, and QOL. The ED offers a teachable moment.

\section{Urban and Rural Differences in the Use of Written Asthma Action Plans}

R. Chooniedass, A. McKillop, T. Lilley-Chan, M. Lilley, S. Huq, J. Liem, A. Becker, Manitoba Institute of Child Health, University of Manitoba, Winnipeg, $M B$

Introduction: Written asthma action plans are central to guideline recommendations for asthma self management. These plans enable patients to adjust their own treatment regimens based on symptoms in order to reduce the incidence of hospitalizations and other morbidity associated with asthma exacerbations. We studied children with asthma in Manitoba and assessed the presence of written asthma action plans by evaluating children in both urban and rural settings. Methods: From among children participating in our Study of Asthma Genes and the Environment (SAGE), born in 1995 in Manitoba, 25 urban and 25 rural children with asthma were randomly selected. They were contacted for a telephone questionnaire to determine whether they had a written asthma action plan and to assess their asthma control. Asthma control parameters were those defined by the Canadian Asthma Consensus Report, 1999. Results: Of the 50 children, only $28 \%$ had a written asthma action plan (11/25 urban vs. $3 / 25$ rural, $p=.01$; OR 5.8, 95\% CI 1.4-24.4). Urban participants had better asthma control based on a positive response to $<2 / 6$ asthma control parameters than rural participants $(614 / 25$ vs. $6 / 25, p=.02$; OR $4.0,95 \%$ CI 1.2 - 13.5). Conclusion: Urban children are more likely to have a written asthma action plan and more likely to have better control of asthma. We believe that there needs to be more emphasis on asthma education especially in rural areas.

\section{Seed Anaphylaxis: A Case Series}

\section{Lori Connors, William Yang, Gina Lacuesta}

Background: Flax (Linum usitatissimum) seeds, sunflower (Helianthus) seeds and other seeds are increasingly used in bread products, including energy and granola bars. Hypersensitivity to these seeds has been infrequently described but important to consider. We report three cases of anaphylaxis to various seeds following ingestion, namely, flax, sunflower and mustard seeds. Methods and Results: Three cases are presented, including clinical course and positive skin-prick testing to commercially available seed extracts. Flax seed exposure through ingestion of an energy bar leads to respiratory and gastrointestinal symptoms of mild anaphylaxis in a 49-year-old woman. Following ingestion of a protein bar containing $97 \%$ sunflower seeds, a 29 - year-old male developed urticaria and angioedema of the throat, which was treated with epinephrine. A 50year-old woman had symptoms of anaphylaxis following ingestion of a hamburger with mustard. She had a previous history of similar symptoms with ingestion of mustard. All three cases suggest type I hypersensitivity reactions to either flax, sunflower or mustard seeds. Conclusion: Flax seeds, mustard seeds and sunflower seeds should be considered as potential allergens. The widespread use of these seeds in energy bars, protein bars and other health food products can make these allergens difficult to identify. Physicians should be aware of the possible presentations of food anaphylaxis and consider foods not traditionally thought to be allergenic.

\section{Interleukin-12 Inhibits Eosinophil Degranulation and Migration but Does Not Promote Eosinophil Apoptosis}

Francis Davoine, Claudine Ferland, Jamila Chakir, Joo Eun Lee, Darryl J. Adamko, Redwan Moqbel, Michel Laviolette, Unité de recherche en pneumologie, Centre de recherche de l'Hôpital Laval, Institut universitaire de cardiologie et de pneumologie de l'Université Laval, Québec, QC; Pulmonary Research Group, Department of Medicine, University of Alberta, Edmonton, $A B$

Background: Animal and human studies demonstrated that interleukin (IL)-12, a Th1 cytokine, reduces blood and bronchial eosinophilia, and airway hyperreactivity. According to current concepts, these effects are mediated through the release of cytokines promoting eosinophil recruitment and activation. However, the presence of IL-12 receptors on eosinophils suggests that IL-12 also acts directly on eosinophils. Hypothesis: We postulated that IL-12 directly modulates eosinophil functions and has the capacity to regulate eosinophil degranulation, migration and survival, in vitro. Methods: Effect of IL-12 on purified human blood eosinophils were evaluated for peroxidase (EPO) release, eotaxin-induced migration through a model of basement membrane (Matrigel ${ }^{\mathrm{m}}$ ), and survival (annexin $\mathrm{V}$-propidium iodide staining and flow cytometry analysis). Results: IL-12 inhibited 50\% of PAF and secretory IgA-induced EPO release $(n=8, p<.001)$. IL-12 also reduced eotaxin-induced migration through Matrigel by $54 \pm 6 \%(n=6, p<.01)$. These effects were not explained by an IL-12 induced impaired viability or apoptosis. Compared to the control medium, IL-12 did not significantly modify viable $(44 \pm 5$ and $56 \pm 8 \%$, control medium and IL-12 respectively, $n=12$, $p=.189)$, apoptotic cell $(29 \pm 5$ and $25 \pm 6 \%$, $p=.161)$ and necrotic cell $(26 \pm 3$ and $18 \pm 4 \%$, $p=.710)$ counts after an $18 \mathrm{~h}$ incubation. Conclusion: Our results demonstrate that IL-12 directly modulates 
eosinophil functions without promoting apoptosis and explain, at least in part, the effects of IL-12 on eosinophils observed in in vivo studies.

\section{Human Eosinophils Express Granzyme B and Perforin: Potential Role in Tumour Killing in Oral Squamous Cancer}

Francis Davoine, Adrian Sim, Tom Wierzbicki, Chris Leong, Lakshmi Puttagunta, Tim McGaw, Donald Yu, Redwan Moqbel, Pulmonary Research Group and Oral Medicine and Pathology, University of Alberta, Edmonton, $A B$

Background: Tumour associated tissue eosinophilia (TATE) characterizes a number of neoplastic conditions, and may have positive prognostic value, particularly in oral squamous carcinoma. However, little is known about the exact role eosinophils play in either carcinogenesis or tumour regression. Granzyme B (GrB) and perforin are two well-characterized antitumor molecules produced by activated $\mathrm{T}$ cells and NK cells. These molecules were detected in murine eosinophils and shown to induce apoptosis in tumour cells but were not yet identified in human eosinophils. Hypothesis: Human eosinophils are specifically recruited to sites of selective tumour growth and play an effector role through cytokine-regulated action of GrB and perforin. Methods: Eosinophils were purified from the peripheral blood of consenting eosinophilic subjects by immunomagnetic negative selection. Cells were probed by RT-PCR for mRNA expression for $\mathrm{GrB}$ and perforin in the presence and absence of IL-2, IL-5, IFN $\gamma$ or IL-12. Flow cytometry of permeabilized eosinophils, with and without the same cytokines, was used to determine the intracellular expression of $\mathrm{GrB}$ protein in these cells. Results: Human eosinophils expressed both mRNA and protein for GrB and perforin. Gene expression for $\mathrm{GrB}$ was induced following overnight incubation with IL-2 and IL-12, but not IL-5 or IFN $\gamma$. FACS analyses revealed that $15 \%$ of unstimulated eosinophils expressed $\mathrm{GrB}$ protein. Cytotoxicity and apoptosis assay using caspase inhibitors and $\mathrm{GrB}$ competitive substrate showed weak contribution of protease-induced apoptosis of both intact and eosinophil lysates on two different cancer cell lines (Jurkat and A594). Conclusion: TATE associated with positive prognosis may be in part due to the capacity of eosinophils to kill target cells in situ by protease-induced apoptosis suggested by their expression of $\mathrm{GrB}$ and perforin. However, since eosinophil lysates exhibited very potent cytotoxic activity, the specific contribution of $\mathrm{GrB}$ and perforin in eosinophil anti-neoplastic activity and the mechanism involved in eosinophilcancer interactions requires further exploration.

\section{Memory Cytokine Responses to Respiratory Viruses in Healthy and Asthmatic Children}

R. Douville, Y. Li, N. Bastien, A. Becker, A. Kozyrskyj, K.T. HayGlass, CIHR National Training Program in Allergy and Asthma Research, Departments of Immunology, Medical Microbiology and Pediatrics/Child Health; University of Manitoba, Canadian Science Center for Human and Animal Health, Department of Community Health Sciences, Faculties of Medicine and Pharmacy, Winnipeg, $M B$

Respiratory syncytial virus (RSV) and Metapneumovirus (MPV) are RNA viruses that commonly infect children. Severe RSV and MPV infections causing bronchiolitis have an epidemiologic association with asthma pathogenesis. They may also be triggers of asthma exacerbation. We hypothesized that these ubiquitous viruses elicit potent recall responses dominated by Th1-biased cytokine production, and that the intensity of such recall cytokine responses associates with current clinical status. Specifically, we propose that asthmatic individuals exhibit heightened immune and clinical responsiveness to these viruses.

We established short-term primary culture systems using peripheral blood mononuclear cells (PBMC) from 8- to 9-year-old children to evaluate (i) the prevalence of virus-specific cytokine recall responses, (ii) the identity of the virus-induced cytokine producing cell subsets, (iii) the hypothesis that different recall responses are evident to respiratory viruses in asthmatic versus non-atopic children. Fresh PBMC from $>60$ children were isolated and cultured with live virus. Supernatants were harvested 6 days later, with the frequency and intensity of type 1 (IFN $\gamma$ CXCL10), type 2 (IL-13), CCL5 and IL10 virus-specific responses quantified by ELISA. Clinical parameters, such as physician-diagnosed asthma, rhinitis and dermatitis in addition to skin prick test and airway hyperresponsiveness measurements were compared with virus-specific cytokine responses. MPV-specific IFN $\gamma$ responses were stronger in asthmatic children compared to non-asthmatic $(p<.05)$. Interestingly, this difference was not apparent in RSV-specific IFN $\gamma$ responses. In summary, these respiratory viruses provide a powerful model for examination of the impact of viral infections in modulating exacerbation of lung inflammation in asthmatic individuals. Support: Camadian Institute of Health Research, Canada Research Chair in Immune Regulation, Tom and Mindel Olenick Award in Immunology, Manitoba Health Research Council. 


\section{IL-17 Counter-Regulates GM-CSF Cytokine- Induced Survival in Human Neutrophils}

Stéphane Dragon, Lianyu Shan, Abdelilah Soussi Gounni, Department of Immunology and National Training Program in Allergy and Asthma, Faculty of Medicine, University of Manitoba, Winnipeg, MB

Interleukin (IL)-17 is a pleiotropic, pro-inflammatory cytokine that has been implicated in many chronic inflammatory and degenerative disorders. Of such, obstructive airway diseases like asthma and chronic obstructive pulmonary disease (COPD) are associated with increased levels of IL-17. IL-17's function links the activation of memory $\mathrm{T}$-lymphocytes to the recruitment and activation of neutrophils to inflamed tissues; however, a direct role for IL-17 on neutrophil function has not been established. In this study, we have evaluated the expression of the IL-17A receptor (IL-17AR) on human peripheral blood neutrophils by RT-PCR and by flow cytometry analysis. We have assessed the rate of IL-17 induced neutrophil apoptosis by annexin-V/propidium iodine (PI) staining and by DNA fragmentation analysis after 6- and 18-hour cultures, respectively. Anti-apoptotic $\mathrm{Mcl}-1$ protein and mRNA levels were detected by Western blot and quantitative $\mathrm{RT}$-PCR, respectively. Bax aggregation was detected by immunocytochemistry and caspase-3/7 activation was assessed fluorometrically. Our results demonstrate human peripheral blood neutrophils express mRNA, intracellular and surface-bound IL-17 receptors. Flow cytometry and cellular morphology assessments demonstrated that recombinant human IL-17 accelerated human neutrophil apoptosis in vitro. Additionally, IL-17 abrogated the anti-apoptotic effect of granulocyte-macrophage colony-stimulating factor (GM-CSF) by specifically reducing Mcl-1 at both the protein and mRNA levels. Bax aggregation ensued concomitantly and caspase- 3 activity increased consequently. Taken together, our findings demonstrate that IL-17 accelerated neutrophil apoptosis and counteracted the survival effects of GM-CSF through a negative feedback loop dependent on the expression of Mcl-1. These results suggest that IL-17 may regulate neutrophilic homeostasis in vivo, and favour the resolution of inflamed tissue by prematurely inducing neutrophil apoptosis. Research funded by the Manitoba Medical Service Foundation and Manitoba Health Research Foundation Grants; A.S. Gounni is supported by a Canadian Institute of Health Research (CIHR) New Investigator Award; S. Dragon is supported by a studentship from the Health Sciences Centre Foundation and by a CIHR National Training Program in Allergy and Asthma.

\section{Syk Kinase in Germ-Free Lung: Any Relationship to the "Hygiene Hypothesis"?}

Florentina Duta, Marina Ulanova, Lakshmi Puttagunta, Daniel Seidel, Ulrich Steinhoff, Alan D. Schreiber, A. Dean Befus, Departments of Medicine, Laboratory Medicine and Pathology, University of Alberta, Edmonton, AB; Department of Immunology, Max Planck Institute of Infection Biology, Berlin, Germany; University of Pennsylvania School of Medicine, Philadelphia, PA, USA

Rationale: Spleen tyrosine kinase (Syk) is a central signaling molecule, best known for its role in IgE receptor activation and initiation of allergic reactions. We previously showed that antisense oligonucleotide (ASO) treatment targeting Syk is an effective anti-inflammatory treatment in a rat asthma model. Given the importance of Syk as a potential target in allergic diseases, we tested whether the regulation of Syk expression might relate to the "hygiene hypothesis," which states that there is an increased prevalence of allergic diseases under reduced microbial exposure. Accordingly, we investigated Syk distribution in tissues of germ-free and conventional mice. Methods: We used immunohistochemistry for protein localization in tissue, Western blot analysis for detection of total Syk protein and detection of its two isoform Syk (L) and (S) and RT-PCR for mRNA expression. Results: We found that Syk kinase is widely expressed in mouse tissues. In the lung, Syk was detected in large and small airway epithelium, Clara cells, type I and II pneumocytes, macrophages, smooth muscle cells and nerves. Both Syk (L) and (S) are present in the lung with differences in their expression between germ-free and conventional mice. Lack of microbial exposure significantly enhanced Syk (S) but not Syk (L) protein expression. Conclusion: Thus because Syk kinase is widely distributed in lung and other mouse tissues, we postulate that Syk kinase is constitutively present in mouse tissues and plays an important role in lung and other organ development and in various physiological functions. Our observation of increased Syk (S) in germ-free mice suggests that the relationship between isoforms of Syk and the "hygiene hypothesis" should be tested further. Funded by CSACI/CAAIF/Merck Frosst, Alberta Heritage Foundation for Medical Research and $\mathrm{NIH}$.

\section{Factors Affecting the Allergic Rhinitis Response to Ragweed Allergen}

Anne K. Ellis, Jodan D. Ratz, Andrew Day, Elizabeth Rafeiro, James H. Day, Queen's University, Kingston, ON; Kingston General Hospital, Kingston, ON

Background: Persons with seasonal allergic rhinitis (SAR) respond to allergen re-exposure differently. This study was designed to determine influences on rate and 
degree of symptom development to controlled ragweed pollen exposure. Methods: Demographics, recent exposure history to household allergens and irritants, as well as Rhinoconjunctivitis Quality of Life Questionnaire (RQLQ) data were obtained from ragweed allergic subjects who also underwent skin-prick testing to selected aeroallergens. Nasal eosinophils were counted. Subjects returned for 3-hour ragweed pollen exposure in the Environmental Exposure Unit (EEU) where a Total Symptom Score (TSS) curve was generated by rating rhinoconjunctivitis symptoms q30 min. A mixed-effects model for repeated measures compared TSS curves between baseline factors. Results: 123 subjects completed the study. Skin test reactivity to ragweed did not correlate with TSS curve generation. Significant differences were noted at 90 min between TSS curves for subjects with positive vs. negative skin test reactivity to dust mite, dog, and grass, as well as subject self-report of symptoms upon dog, cat, and other animal exposure. The TSS curves generated in these groups showed general trends towards the entire 3-hr pollen exposure being different. Visual analogue scale ratings of SAR symptoms during both ragweed and grass seasons and RQLQ scores were also positively associated with TSS curves. No other associations were detected. Conclusion: This study indicates a relationship between the rate and degree of symptom development to controlled ragweed exposure and immediate skin test reactivity to dust mite, animals, and grass pollen. Symptom development also correlated with self-reported symptoms to animals, seasonal grass and ragweed, as well as rhinitis-specific quality of life. No associations were shown with latephase response, nasal eosinophils or degree of skin test reactivity to ragweed.

\section{The Allergic Rhinitis Experience: A Self-Reported Patient Evaluation of Symptomatology and Medication Use During Ragweed Season}

Anne K. Ellis, Jodan D. Ratz, Matthew J. Heffer, James H. Day, Queen's University, Kingston, ON

Background: Medication utilization patterns of patients suffering from seasonal allergic rhinitis (SAR) are not well documented, and although many anti-allergic medications are prescribed for daily use, their actual usage is known to be quite variable. Methods: 1821 subjects with positive ragweed skin tests were mailed a survey during the third week of ragweed season, soliciting the nature and severity of SAR symptoms, usage patterns and reasons for choice of anti-allergic medication. Results: 550 subjects completed the survey (30.2\%). The prevalence of symptoms were, in decreasing order: sneezing (91.5\%), runny nose $(82.4 \%)$, itchy/gritty eyes $(80.4 \%)$, stuffiness (78.5\%), itchy nose $(71.3 \%)$, watery eyes $(64.7 \%)$, itchy palate/throat $(56.9 \%)$, post-nasal drip $(55.6 \%)$, red/ burning eyes $(49.1 \%)$, headache $(36.5 \%)$, itchy ears $(34.0 \%)$, cough $(30.0 \%)$, shortness of breath (15.7\%), and wheeze (15.5\%). SAR patients used antihistamines most frequently (94.7\%), followed by decongestants (63.1\%), combination (antihistamine/decongestant) products $(52.0 \%)$, and intranasal corticosteroids (42.5\%). Medications were mostly taken intermittently rather than daily (antihistamines 68.0\%; nasal corticosteroids 71.8\%). Conclusion: Aconstellation of nasal symptoms was the most common seasonal allergic manifestations, followed by ocular, palatal and ear irritation. Antihistamines were the most frequently used medication to treat symptoms, succeeded by decongestants (alone or in combination). A significant proportion of subjects took their allergy medication, including nasal corticosteroids, intermittently rather than regularly, underscoring the relevance of single-dose evaluations of drug efficacy.

\section{Ability of Elementary School Teachers to Use Epi-pens}

David Fischer, University of Western Ontario, London, ON

This prospective study documents the ability of teachers to emergently administer Epi-pens in the public school system. Method: 100 prospective elementary (K-8) school teachers seen in an allergy clinic were questioned about the presence of anaphylactic children in their schools, their preparedness level, and then asked to administer an Epi-pen trainer without reading the instructions. Results: $87 \%$ of the teachers had children in their school who carried Epi-pens (directly responsible in $82 \%$ of cases). $76 \%$ of the teachers had received some workplace Epi-pen training. None of them had ever used a real Epi-pen. 28\% of the teachers either carried an Epipen themselves or had a 1 relative with one. A further $6 \%$ had taken extra first aid training. 6/28 teachers (21\%) owning Epi-pens had had no training. 23\% would have administered it in a way dangerous to them or the child. A further 30\% aggressively grabbed the needle end which may have activated it. $10 \%$ would have immediately pulled the needle out after activating it. The pass rate for all comers was $12 \%$. A further $10 \%$ made some errors but would likely have worked without significant harm (total of $22 \%$ ). Of the 12 subjects who passed, only 3 did not have previous specialized knowledge. The pass rate for those without prior specialized knowledge was $3 / 66$ $(4.5 \%)$ or $8 / 66$ (12\%) if equivocals were considered passes. With specialized knowledge, 9/34 (26\%) passed, rising to $14 / 34$ (41\%) if equivocal responses were included. Only one person with no prior training passed. Conclusion: Although some teaching programs in Epi-pen use exist, teachers, especially those without prior specialized knowledge, are still lacking the necessary skills to provide this potentially life-saving therapy. 


\section{SNARE Isoform Expression in Cytotoxic T-Cells and Natural Killer Cells}

D.L. Garofoli, S.O. Odemuyiwa, M.R. Logan, K. Lo, R.C. Bleackley, R. Moqbel, Pulmonary Research Group and Department of Biochemistry, University of Alberta, Edmonton, $A B$

Rationale: Cytotoxic T-cells (CTL, CD8+ cells) kill altered self-cells, including virus-infected or tumour cells, through granzyme B (GrB)-mediated apoptosis. Although the mechanism of GrB-mediated killing of target cells is well characterized, the intracellular machinery involved in CTL cytotoxic granule secretion of GrB is uncertain. Secretory cell exocytosis is dependent on interactions between SNARE (soluble NSF attachment protein receptor) proteins on vesicular membrane ( $\mathrm{v}$ SNAREs) and target plasma membrane (t-SNARE). We hypothesized that SNARE proteins are involved in the regulated release of GrB stored in CTLs and natural killer (NK) cells. Methods: Specific primer sets and RTPCR were used to determine SNARE mRNA expression in human CD8+ lymphocytes and YT-Indy, a human NK cell line. Western blot analysis and immunocytochemistry, using specific antibodies, confirmed SNARE protein expression and determine the localization of selected SNARE proteins. Results: CTLs and YT-Indy express mRNA encoding for the v-SNAREs, VAMP 1, 2, $3,7,8$, and the t-SNAREs, syntaxin 3, 4, 6, and SNAP23. We confirmed expression of SNAP-23, syntaxin-4, syntaxin-6, VAMP-7 and VAMP-8 in CTLs and YTIndy by Western blot analysis. VAMP-8 protein is not expressed in YT-Indy. Confocal microscopy supported data of Western blot analysis in both CTLs and YTIndy. Conclusion: Our data suggest a unique profile of SNARE proteins are involved in exocytosis of cytotoxic granules from CTLs and YT-Indy. Supported by Canadian Institutes of Health Research.

\section{The lle117Thr Polymorphism of the GM-CSF Gene is Associated with Atopic Dermatitis in a Prospective Study}

J.-Q. He, M. Chan-Yeung, A.B. Becker, H. Dimich-Ward, A.C. Ferguson, J. Manfreda, W.T. Watson, P.D. Paré, A.J. Sandford, James Hogg iCAPTURE Centre for Cardiovascular and Pulmonary Research, St. Paul's Hospital, University of British Columbia, Vancouver, BC; Occupational and Environmental Lung Diseases Unit, Department of Medicine, University of British Columbia, BC; Section of Allergy and Clinical Immunology, Department of Pediatrics, University of Manitoba, Winnipeg, $M B$

Background: Genetic polymorphisms of the GM-CSF gene have been reported in case control studies as important genetic markers predicting an individual's predisposition to atopic dermatitis and other allergic diseases. We previously reported this association in at-risk children at 1 to 2 years of age. We hypothesized that this association will be consistent for those children at 7 years old. Methods: This prospective study cohort contained 215 white children at high risk of developing atopy and atopic disorders because at least one firstdegree relative had asthma or two first-degree relatives had other allergic diseases. We investigated whether the Ile117Thr polymorphism of the GM-CSF gene was associated with atopic dermatitis and other related phenotypes such as atopy, allergic rhinitis and asthma. Results: In 215 white children, the prevalence of atopic dermatitis at 7 years of age was significantly increased in children with the 117Ile allele $(16 / 93=17.2 \%)$ compared with children without the 117 Ile allele $(9 / 122=7.4 \%)$. The relative risk (RR) for atopic dermatitis for children with the 117Ile allele compared to children without the 117Ile allele was 2.3 (95\% CI 1.1-5.0, $p=.026)$. After adjusting for confounding factors such as sex and intervention group, the RR was 2.8 (95\% CI 1.2-7.2, $p=.021)$. No association was found for the Ile117Thr polymorphism of the GM-CSF gene with asthma and other allergic diseases at 7 years of age. Conclusion: Our data further support that the 117Ile allele of the GMCSF gene is a risk factor for the development of atopic dermatitis at 7 years of age. Supported by the Canadian Institutes of Health Research.

\section{Non-allergic Children Demonstrate Th2 Responses to Peanut}

Sherry Hebert, Anita L. Kozyrskyj, Allan B. Becker, A. Wesley Burks, Kent T. HayGlass, CIHR National Training Program in Allergy and Asthma Research, Departments of Immunology, Community Health Sciences, and Pediatrics/Child Health, University of Manitoba, Winnipeg, MB, Canada and Department of Pediatrics, Duke University, Raleigh NC, USA

Introduction: Despite its prevalence, little is known of the role of $\mathrm{T}$ cells in the development and maintenance of peanut allergy. Previous investigations into the role of cytokines and chemokines in various atopic diseases suggest that Th1 responses are protective. However, the presence and intensity of Th1 responses to peanut antigens by non-allergic individuals is still controversial. Methods: We have used a primary PBMC culture system to investigate the expression of various cytokines and chemokines by non-allergic children following stimulation with whole peanut extract. Analysis of supernatants was carried out by ELISA. These children were further characterized according to the presence of positive or negative skin tests to peanut (sensitized vs. non-sensitized), the presence of other atopic diseases, gender, and place of residence. Results: Among both groups of nonallergic children (peanut responders and non-responders), Th2 responses predominated (IL-5, IL-13, CCL17, 
and CCL22), with a lack of significant Th1 cytokine or chemokine production (IFN- $\gamma$ and CXCL10). The frequency and strength of these Th2 responses were greater for sensitized children. They are dependent on $\mathrm{CD} 4^{+} \mathrm{T}$ cells, requiring antigen presentation through HLA-DR, and co-stimulation via CD86. IL-10 production was detected in both groups of non-allergic children and may therefore play a role in preventing the development of food allergy. Finally, we did not observe any influence of gender, place of residence, or other atopic disease on the responses of these children to peanut antigens. Conclusion: Our analysis of peanut-specific responses by non-allergic children have demonstrated that (i) non-allergic children produce Th2, not Th1, responses to peanut allergens, (ii) sensitized children demonstrate increased Th2 responsiveness to peanut, (iii) stimulation with peanut results in the production of IL-10, which may contribute to protection from the development of allergy, and (iv) these responses in nonallergic children do not appear to be affected by gender, place of residence or the presence of other atopic diseases. (Supported by CIHR, NSERC Studentship, National Training Program in Allergy and Asthma Research, Canada Research Chair in Immune Regulation)

\section{Inactivated Bacterial Exposure in Early Life Has Long-term Inhibitory Effect on Allergic Responses Possibly through Modulating Dentritic Cell (DC) Function}

L. Jiao, X. Yang, Department of Medical Microbiology, Faculty of Medicine, University of Manitoba, Winnipeg, $M B$

Introduction: Our previous studies have shown that chlamydial infection in adult mice can inhibit the development of allergic reaction induced by allergens such as ragweed and ovalbumin (OVA). The aim of the present study was to determine the effect and mechanism of exposure to chlamydial products in early life on the development of allergic responses when the mice become adults. Methods: Newborn C57BL/6 mice were immunized intraperitoneally (i.p.) or subcutaneously (s.c) with various doses $\left(1 \times 10^{4}-1 \times\right.$ $10^{7}$ inclusion forming units) of UV-killed Chlamydia trachomatis mouse pneumonitis (MoPn) at different ages (5, 14, 21 and 28 days of age) and sensitized with OVA in alum one month later. The mice were challenged intranasally with OVA at two weeks after sensitization and tested for pulmonary allergic inflammation and immune reaction. DCs were isolated from UVkilled MoPn-treated (iDc) and mock-treated mice (nDc) at different time points (1 week or 4 weeks after treatment of UV-killed MoPn) and examined for surface markers by flow cytometry and RT-PCR, and cytokines production by ELISA. To further investigate the role played by DCs in inactivated-MoPn mediated inhibition of allergic responses, iDC and $\mathrm{nDC}$ were adoptively transferred, respectively, to syngeneic naive mice $\left(5 \times 10^{6}\right.$ cells/mouse) intravenously. Two groups of naïve mice were sensitized with OVA in alum at two hours after the adoptive transfer and challenged with OVA at two weeks after sensitization and the effect of DC transfer on allergic reaction induced by allergen exposure was examined. The role of DCs in directing $\mathrm{CD}^{+} \mathrm{T}$ cell differentiation is investigated by measuring cytokines production in $\mathrm{DC}-\mathrm{CD} 4^{+} \mathrm{T}$ co-culture system in vitro. Results: Our results showed that vaccination with inactivated MoPn significantly inhibited airway eosinophilia, VCAM-1 and ICAM-1 expression, and mucus production induced by OVA exposure. This is associated with a significant decrease in allergen-driven Th2 cytokine production by spleen and draining lymph node $\mathrm{CD}^{4} \mathrm{~T}$ cells. Surface markers analysis showed that DCs from inactivated bacteria treated mice (iDc) exhibited elevated CD8a, CD80, CD86 and MHC-II molecules, and produced higher levels of IL-12 and IL-10 compared to DC from the mock treated group ( $\mathrm{nDc}$ ). IDCs drive naïve $\mathrm{CD}^{+} \mathrm{T}$ from Th2-dominant to Th1- dominant immune response, and adoptive transfer of $\mathrm{iDC}$, but not $\mathrm{nDC}$, inhibited allergic reaction induced by allergen exposure in vivo. Conclusions: Our results indicate that early exposure to bacterial products have a long-term inhibitory effect on the development of allergic responses induced by allergen possibly through altering the function of DCs.

\section{Effectiveness of Desloratadine in the Treatment of Seasonal Allergic Rhinitis (SAR) in an Open, "Real-World" Setting: Results from the Partners in Allergy Control and Therapy (PACT) Study}

Paul K. Keith, George Luciuk, McMaster University, Hamilton Health Sciences Centre, Hamilton, ON; Canada and British Columbia Children's Hospital, Richmond Hospital, Richmond, BC

Background: In clinical trials, the long-acting, nonsedating oral antihistamine desloratadine relieves nasal congestion as well as the non-nasal symptoms of allergic rhinitis. Previous reports have indicated that $69 \%$ of Canadian adults with allergic rhinitis cite nasal congestion as their most bothersome symptom. The aim of the PACT study-a large, open-label Canadian study in a "real-life" setting-was to assess the effectiveness of desloratadine in a community setting. Methods: 6,829 subjects with SAR ( $>12$ years) were treated during the spring time with desloratadine (one $5 \mathrm{mg}$ tablet daily) for 7 days. The treating physician completed a questionnaire with the patient prior to dispensing 
treatment and after 7 days of treatment. Results: After 7 days, overall symptoms were significantly less than at baseline $(p<.0001)$, with a $55 \%$ drop in symptom severity. All individual symptoms improved, including nasal stuffiness/congestion, nasal discharge, nasal itching, sneezing, itching of the palate, coughing and eye symptoms $(p<.0001$ for all symptoms). $89 \%$ of subjects experienced improvement in overall allergy symptoms and $88 \%$ had relief of nasal congestion. Of the $50 \%$ of subjects who were taking concomitant medications (most commonly nasal corticosteroids), 30\% had moderate-to-severe baseline symptoms. The two-way mixed model ANOVA showed a statistically significant incremental benefit when combining desloratadine and a nasal corticosteroid $(p=.0002)$. All patients, whether receiving nasal corticosteroids or not, had significant symptom relief, including improved nasal congestion $(p<.0001)$. Conclusions: Desloratadine treatment significantly reduced the severity of all symptoms by $55-70 \% .88 \%$ of subjects experienced relief of nasal congestion after only one dose. The concomitant use of desloratadine and a nasal corticosteroid in patients with moderate-to-severe baseline symptoms showed an incremental benefit for relieving nasal congestion. These findings indicate that desloratadine is well suited as a first-line choice for the treatment of SAR in a community setting, including those patients experiencing nasal congestion. Funding: Schering Canada.

\section{Effectiveness of NASONEX ${ }^{\circledR}$ (Mometasone Furoate) Nasal Spray in the SHARE (Symptom History and Report on Effectiveness) Program}

Harold Kim (Assistant clinical professor, Faculty of Medicine, McMaster University)

Objectives: To investigate patient satisfaction and efficacy of regular use of Nasonex in allergic rhinitis, with and without concomitant use of antihistamines. Methods: Adult patients with moderate or severe allergic rhinitis were enrolled by 435 physicians across Canada. The patients were treated with Nasonex $50 \mathrm{mcg} / \mathrm{spray}$ two sprays in each nostril od for 14 days. Patients could take other allergy medications during the study. All subjects were asked to complete a symptom and product appreciation questionnaire. Results: Questionnaires were completed by 1,242 of 2,486 patients. Females comprised $60.9 \%$ of subjects. At the start of the study, $48.9 \%$ were taking an oral antihistamine. Patients reported reductions in all nine symptoms (limitation of activities, sneezing, stuffy nose, runny nose, watery eyes, itchy eyes, sleep impairment, fatigue, irritability) of $44 \%$ to $60 \%$ ( $p<.0001$ for all symptoms). Appreciation of six product characteristics (effectiveness, symptom relief, ease of use, fine mist, plastic bottle, pleasant to take) was rated from $73 \%$ to $85 \%$. Both simple and covariate analysis indicated a significant benefit of combination therapy of Nasonex with a non-sedating antihistamine compared to Nasonex alone. Improvement with concomitant use of antihistamine was reported by $84.9 \%$ of patients. Also, daily use of Nasonex was significantly better than intermittent use. Conclusion: Patient symptom reduction and satisfaction are both very good for Nasonex. Concomitant use of antihistamines may further improve benefits, particularly in more severe cases.

\section{Identifying Childhood Asthma from Health Care Database Records: Is It Valid?}

Anita L. Kozyrskyj, Allan B. Becker, Dept. of Community Health Sciences, Department of Pediatrics and Child Health, and Faculty of Pharmacy, University of Manitoba, Winnipeg, $M B$

Rationale: To determine the validity of a health care database definition of asthma for future use in population- based studies. Methods: Using health care database records, asthma at age 7 to 8 years was identified in a 1995 Manitoba birth cohort on the basis of physician visits or hospitalizations for asthma (ICD9 code 493), or receipt of asthma prescription medications (eg, $\beta$-agonists, inhaled corticosteroids/cromones, montelukast). A case-control sample of cohort children was recruited in 2003/04. The positive predictive value (PPV) and Youden's Index for several variations of the database definition in the preceding year were determined in case-control children against a selected gold standard: asthma diagnosis by a pediatric allergist. Results: Clinical assessment, PC20 methacholine and symptom data were available for 556 children aged 8 to 9 years in the case-control sample. $58 \%$ of children aged 8 had prescription medications or health care visit for asthma in 2002, 68\% had allergist-diagnosed asthma, $74 \%$ had a positive methacholine challenge test $(\mathrm{PC} 20<8 \mathrm{mg} / \mathrm{mL})$ and $55 \%$ had recent wheezing. $78 \%$ of children with allergist-diagnosed asthma had a positive methacholine challenge test and $72 \%$ wheezed in the last 12 months. Using allergist diagnosis as the gold standard, the sensitivity of a database definition based on at least one asthma health care visit or prescription drug was $75 \%$ and the specificity was $79 \%$. The PPV value increased from $88 \%$ to $93 \%$ and the Youden's Index decreased from 0.54 to 0.33 , as the number of asthma health care visits and prescription drugs increased in the database definition. Findings were similar for children aged 9 years. Conclusion: Ahealth care database definition of asthma based on prescription and health care use is a valid method to identify asthma, but the selection of a specific definition is dependent on the purpose of the study. 


\section{Asthma Phenotype and Early Life Exposure: Differences between First Nations and Non First Nations Children}

Anita L. Kozyrskyj, Joel J. Liem, Allan B. Becker, Dept. of Community Health Sciences, Department of Pediatrics and Child Health, and Faculty of Pharmacy, University of Manitoba, Winnipeg, $M B$

Rationale: To determine the asthma phenotype and early life exposures in First Nations (FN) and non First Nations community children. Methods: Anested casecontrol study of children with and without asthma was conducted in a 1995 Manitoba birth cohort. The association between pediatric allergist-diagnosed asthma, phenotype and early childhood exposures to tobacco smoke, pets and mold was determined in FN and non FN community children, using chi square at a significance level of $p<.05$. Results: 721 children aged 8 to 10 years were recruited for the case-control study. 82 children were born and currently living in a FN community, 7 were born in a FN community, but no longer lived there, and 632 were all other Manitoba children. Birth exposures to mold (54\%) and tobacco smoke (76\%) were 1.5 to 2 times higher in FN community than other Manitoba children. Percentage exposure to cats and dogs at birth was similar among all children. FN community children with allergist diagnosed-asthma were significantly more likely than those with no diagnosed asthma to have at least one positive skin test $(50 \%)$. No differences were observed for bronchial hyper-responsiveness or early life exposure to tobacco smoke, pets or mold in FN community children with asthma compared to those without asthma. Children with asthma not living in a FN community were also significantly more likely than those without asthma to have at least one positive skin test (66\%), but were significantly more likely to have bronchial hyper-responsiveness (methacholine PC20 $<8 \mathrm{mg} / \mathrm{mL}, 79 \%$ ) and to be exposed to mold $(41 \%)$ and to tobacco smoke $(37 \%)$ at birth. Conclusions: Asthma in FN community children is less likely to be associated with bronchial hyper-responsiveness than other Manitoba children. Despite high birth exposures to tobacco and mold, no associations with asthma were reported for FN community children.

\section{Premature Children, Gender, and the Risk of Wheezing}

J.J. Liem, A.L. Kozyrskyj, A.B. Becker, Department of Community Health Sciences, Department of Pediatrics and Child Health, and Faculty of Pharmacy, Universioty of Manitoba, Winnipeg, MB

Rationale: Prepubertal males have a higher prevalence of asthma compared to females. We sought to determine whether premature males have this same predisposition for wheezing when compared to premature females. Methods: The Manitoba Health Services
Insurance Plan (MHSIP) database is a population-based, health care administrative and prescription database. It has records of every child born in 1995 and subsequent utilization of the provincial health care system. The number of children diagnosed with a wheezing syndrome (defined as hospital/physician visit ICD-9 code of 466 [acute bronchitis and bronchiolitis], 490 [bronchitis not specified], 491 [chronic bronchitis], 493 [asthma], or a prescription for an asthma medication) was obtained. The relative risks of wheezing in premature males compared to premature females was determined up to 7 years of age. Results: 13,980 children were born in 1995 and are currently living in the province of Manitoba. In comparison to their female counterparts, the relative risk (RR) of a wheezing syndrome in a premature male born $<32$ weeks gestational age $(\mathrm{GA})(n=118)$ was only statistically significant at age 1 year $(\mathrm{RR}=1.54[\mathrm{CI}=$ 1.07-2.21]). RR for premature males compared to females born at 32 to 37 weeks GA $(n=763)$ was statistically significant at ages $1(\mathrm{RR}=1.31[\mathrm{CI}=1.1-1.56])$ and $2(\mathrm{RR}=1.32[\mathrm{CI}=1.05-1.67])$. Males born at term (>37 wks GA) had statistically significantly higher relative risks for a wheezing syndrome for all 7 years of life when compared to their female counterparts. Conclusion: Males born at term are more likely to have wheezing episodes up to age 7 when compared to females born at term. Beyond the first year of life, premature males do not have a similar predisposition when compared to premature females.

\section{Differential Toll-Like Receptor 4 (TLR4) and TLR2 Responsiveness Is Revealed in Allergic Asthmatic vs Healthy Children Using Threshold Levels of TLR Stimulation}

Yuriy Lissitsyn, Alex Silaghi, Allan B. Becker, Steven Jones, Anita Kozyrskyj, Kent T. HayGlass, Departments of Immunology, Pediatrics/Child Health, and Faculties of Medicine and Pharmacy, University of Manitoba, Winnipeg, $M B$

Introduction: Toll-like receptors are a major family of pattern-recognition receptors that recognize conserved pathogen-associated molecular patterns. They play a key role in initiating innate immunity, and in regulating the nature of the adaptive immune response that subsequently develops. TLR responsiveness in different individuals may influence their likelihood of exhibiting allergic diseases such as asthma. The contributions of TLR function to human asthma remain unknown. We hypothesize that functional responsiveness to TLR stimulation by physiologically relevant ligands differs in asthmatic and healthy children. We previously established highly sensitive experimental systems using "threshold" doses of TLR ligands rather than the typically used "maximal stimulation" concentrations to test 
our hypothesis. This novel approach provides greatly enhanced sensitivity to detect differences in responsiveness. Methods: PBMC obtained from 15 allergic asthmatic and 15 healthy control 7 to 8 year old children were stimulated with the following TLR ligands: lipopolysaccharide (LPS)/TLR4, or peptidoglycan (PGN)/TLR2 at "threshold" and "optimal" doses for $24 \mathrm{~h}$. This time point was chosen on the basis of kinetic studies. Levels of pro-inflammatory (IL-1beta, TNF-alpha) and antiinflammatory (IL-10) cytokines produced by these children were measured by ELISA. Results: Dose-response titration studies reveal much more heterogeneity in cytokine production among individuals when threshold doses of LPS or PGN are examined than when typical, maximal stimulation conditions are used. Experiments using blocking antibodies and testing of TLR stimulation by assessing NF- $\kappa$ B activation in HEK293 cells transfected with either TLR4-MD2-CD14 or TLR2 confirm the specificity of these TLR ligands. Healthy control children exhibit markedly increased IL-1beta and TNFalpha synthesis relative to asthmatics upon stimulation with threshold dose of LPS $(0.5 \mathrm{ng} / \mathrm{mL})$ or PGN $(0.1 \mathrm{ug} / \mathrm{mL})$. Conversely, in response to threshold stimulation with LPS or PGN, IL-10 production is elevated among asthmatic children. Under conditions of maximal pharmacologic stimulation, we did not observe any differences between the groups. Conclusion: Use of threshold concentrations of TLR4 and TLR2 ligands, intended to more closely approximate physiologic stimulation, reveal striking differences in cytokine responses between asthmatic and control children. In contrast, commonly used maximal concentrations of LPS and PGN elicit strong, indistinguishable patterns of cytokine production. The finding of more robust pro-inflammatory cytokine responses to TLR stimulation in healthy children favors a Th1- biased "protective" adaptive immunity. Increased production of anti-inflammatory IL-10 in asthmatic children could represent a homeostatic mechanism in an attempt to control allergen-specific inflammation. Thus, this strategy of using threshold levels of TLR stimulation provides markedly increased sensitivity to reveal functional alterations in TLR responsiveness that associate with clinical status. Research support: SAGE/CIHR; CRC Chair Program.

\section{Immunization with an Interleukin 13 Vaccine Downregulates Allergic Airway Inflammation} Yanbing Ma, Kent T. HayGlass, Srinivasan G, Allan B. Becker, Zhikang Peng, Department of Pediatrics and Child Health and Dept. of Immunology, University of Manitoba, Winnipeg, $M B$

Background: Interleukin (IL)-3 plays an important role in the initiation and development of allergic asthma. We have developed human and mouse IL-13 peptide-based vaccines, chimeric hepatitis $\mathrm{B}$ core antigens ( $\mathrm{HBcAg}$ ) containing IL-13 peptides, which present as virus-like particles and induce high titers of auto-antibodies against IL-13. Objective: We wanted to test the in vivo effect of administration of a mouse IL-13 vaccine in the downregulation of airway allergic responses in asthmatic mice. Methods: Mice were immunized with a mouse IL13 vaccine three times at two-week intervals. Mice immunized with the native $\mathrm{HBcAg}$ served as controls. One week after the final immunization, the mice were sensitized using 2 protocols: two intraperitoneal injections with ovalbumin precipitated in alum at a twoweek interval and intraperitoneal injections with ovalbumin without adjuvant twice per week for 5 weeks. One week after the last sensitization, airway inflammation was induced by intranasal administration of ovalbumin. Two days later, methacholine-induced airway hyperresponsiveness was measured and four days later, bronchoalveolar lavage fluids (BALF) and serum samples were obtained. BALF eosinophils were counted. Cytokine and IgE levels were measured by ELISA. Results: Mice immunized with the vaccine produced high titers of IgG antibodies to IL-13. Eosinophils, mean levels of IL-13 and IL-5 in BALF and serum ovalbumin-specific IgE were significantly reduced in the vaccinated group when compared to the controls. Methacholine-induced airway hyperresponsiveness was also significantly reduced in the vaccinated group. No significant changes were found in the mean levels of IL-4, IFN $\gamma$, and IL-12 between the two groups. Conclusion: Administration of IL-13 vaccine elicited high titers of antibodies to IL-13, leading to a decrease of airway allergic responses in asthmatic mice. This strategy may provide a new therapeutic approach in the treatment of asthma.

\section{Molecular and Ultrastructural Analysis of Piecemeal Degranulation in Neutrophil Azurophilic Granules}

Salahaddin Mahmudi-Azer, Furquan Shaheen, Preet Bubra, Setareh Daneshmend, Mohsen Mousavioun, Peter D Paré, James Hogg iCAPTURE Centre, St. Paul's Hospital, Department of Medicine, University of British Columbia, Vancouver, BC

Neutrophil azurophilic granules store a number of potent proteases known to be central to host defense and also acute and chronic inflammatory responses. Following cell activation these mediators are released to extracellular space where they cause tissue degradation and damage through their proteolytic activity. Molecular mechanisms by which mediators pre-stored in azurophilic granules are mobilized and released to extracellular space remain unknown. In our current study we used a number of complementary techniques including confocal immunofluorescence microscopy, subcellular fractionation, flowcytometry 
analysis, Western blot analysis and electron microscopy to examine the molecular mechanism of selective mediator release in neutrophil azurophilic granules. Our data indicate that mediator mobilization and release from azurophilic granules following cell activation is selective and while cell activation leads to mobilization and release of elastase from azurophilic granules, the MPO content of these granules remains unaltered. The selective mediator mobilization from azurophilic granules is associated with translocation of CD63 and Hck from azurophilic granules to the cell surface. The selective translocation of mediators from azurophilic granules following cell activation is morphologically highlighted by budding of small vesicles from azurophilic granules and also reduced granule size and density. This is the first study of agonist induced selective mediator release (piecemeal degranulation) from neutrophil azurophilic granules which may imply the involvement of CD63, Hck, and $\beta$-arrestin- 1 in these processes. This research was supported by research grants from the American Thoracic Society and Canadian Institute of Health Research.

\section{Exportin 1 Regulates the IL-8 Induced Mediator Release from Neutrophil Azurophilic Granules} Salahaddin Mahmudi-Azer, Furquan Shaheen, Preet Bubra, Kazuyuki Nishimura, Setareh Daneshmend, Mohsen Mousavioun, Peter D Paré, James Hogg iCAPTURE Centre, St. Paul's Hospital, Department of Medicine, University of British Columbia, Vancouver, BC

Mediator mobilization and release from neutrophil azurophilic granules is central to neutrophil bactericidal activity and innate immune response. Agonist activation of CXCR1 on the neutrophil surface is known to induce formation of a $\beta$-arrestin-Hck complex followed by translocation of the complex to azurophilic granules and mediator release from azurophilic granules. The molecular mechanism of intracellular trafficking of $\beta$-arrestin and Hck and formation of their complex remain largely unknown. In this study, we used a number of different techniques including immunofluorescent staining, confocal laser scanning microscopy (CLSM), sub-cellular fractions, flowcytometric analysis, immunoprecipitation, Western blot analysis, and mediator release assays to examine the precise role of exportin 1 in the association of Hck and $\beta$ arrestin and their subcellular redistribution following CXCR1 activation. Here we show that exportin 1 regulated the intracellular trafficking and molecular association among $\beta$-arrestin 1 , Hck and CXCR1. While $\beta$-arrestin 1 seems to be critical in mediator release from neutrophil azurophilic granules, its role in CXCR1 internalization is less prominent. Thus we propose a novel regulatory role for exportin 1 in regulation of intracellular trafficking of Hck and $\beta$-arrestin 1 and also a differential role for $\beta$ arrestin 1 and $\beta$-arrestin 2 in processes associated with neutrophil activation and exocytosis. This research was supported by research grants from the American Thoracic Society and Canadian Institute of Health Research.

\section{Enhanced Fetal Growth and Allergic Diseases in a Birth Cohort Study of BAMSE}

Xiao-Mei Mai, Catarina Almqvist, Lennart Nilsson, Magnus Wickman, Department of Molecular and Clinical Medicine, Division of Pediatrics, Linköping University, Linköping, Sweden; Department of Occupational and Environmental Health, Karolinska Hospital, Stockholm, Sweden; Woolcock Institute of Medical Research, Sydney, Australia

Background: There is a trend in increasing fetal growth in affluent countries, but the relationship between enhanced fetal growth and allergic diseases is not conclusive. Objective: We aimed to assess high birth weight or large birth length in relation to allergic diseases at 4 years, taking childhood overweight into consideration. Methods: A number of 2,869 children from a birth cohort in Stockholm were included (BAMSE). The included children must meet two requirements: the parents had answered all the questionnaires at 2 months, 1 year, 2 and 4 years of age on environmental factors and allergic symptoms, and the children had participated in clinical examination at age 4 including the measurement of body weight and height. Sensitization at 4 years was defined as a positive Phadiatop. Perinatal data were received from the Child Care Health Centers. Birth weight and length, and body mass index (BMI) at age 4 were categorized into three groups according to the 10th and 90th percentiles, respectively. Results: After the adjustment for birth weight, BMI and other potential confounders, birth length $\geq 90$ th percentile was inversely associated with any wheeze at 4 years (OR 0.64, 95\% CI 0.44-0.92) and late-onset wheeze (at least one episode at age 4 but no episode up to age 2) (OR $0.40,95 \% \mathrm{CI}$ $0.21-0.77)$. The relationships were obvious in the children without sensitization at age 4 . Body mass index $\geq 90$ th percentile at age 4 was positively associated with early transient wheeze in nonsensitized children (OR 1.99, 95\% CI 1.22-3.24). Conclusion: Enhanced fetal growth with large birth length may play a protective role in non-atopic late-onset wheeze.

\section{Selective Ig-A Deficiency and Mycobacterium kansasii Infection}

A.D. Moore, B. Ominsky, M. Shochet, S.L. Jacobs, ENTAA CARE, PA, Glen Burnie, MD, USA, Lung Associates of Anne Arundle County, Glen Burnie, MD, USA

Introduction: Selective IgA deficiency is the most common immunodeficiency, observed in up to 1 of 400 blood donors. Mycobacterium kansasii is an atypical mycobacterium known to cause pulmonary infection in 
both immunocompetent and immunocompromised individuals. Case: A 36-year-old mother of two presented with a 3-year-history of a chronic productive cough, post-nasal drainage and shortness of breath on exertion. After multiple treatments for recurrent bronchitis and sinusitis, serological testing revealed the presence of selective IgA deficiency. A chest $\mathrm{x}$-ray subsequently revealed a right upper lobe cavitary pneumonia. The patient was found to have a PPD (purified protein derivative, or tuberculin test) of $20 \mathrm{~mm}$ and sputum specimens demonstrated acid-fast organisms. After initial treatment with quadruple antibiotic therapy for presumptive tuberculosis (isoniazid, rifampin, ethambutol, pyrazinamide), nucleic acid testing and cultures implicated Mycobacterium kansasii as the relevant organism. An additional immune work-up, including testing for HIV, was negative. The patient has been maintained on isoniazid, rifampin and ethambutol with significant clinical improvement. After family screening, a brother and one of two daughters were also found to have selective IgA deficiency. Discussion: Mycobacterium kansasii is a slow growing Mycobacterium that can cause pulmonary infection in patients with predisposing lung disease, although up to $40 \%$ of patients are immunocompetent and have no predisposing condition. Such infection has not previously been described in patients with isolated IgA deficiency. This case highlights the importance of vigilance for immunodeficiencies in adults with frequent upper and lower respiratory infections, the need for prompt investigation of recurrent symptoms, and the utility of screening the families of affected individuals for immunodeficiency.

\section{Production of Nitric Oxide by Mast Cells from Different Rat Strains}

Samira Muñoz, Marcelo Marcet-Palacios, A.D. Befus, Pulmonary Research Group, Department of Medicine; University of Alberta, Edmonton, $A B$

Despite abundant evidence that mast cells (MC) produce nitric oxide (NO), controversy remains because some studies have been unable to detect production of $\mathrm{NO}$ in MC. We postulated that this reflects different strains of rats used in the conflicting studies. Thus, we compared NO production in peritoneal MC isolated from Sprague Dawley (SD) and Brown Norway (BN) rats, and investigated some of the mechanisms that might underlie the differences observed. Parallel experiments using Sprague Dawley (SD) and Brown Norway (BN) MC were done. Unstimulated MC from both strains spontaneously produced a low amount of NO. Stimulation with IFN $\gamma$ or IFN $\alpha / \beta$ (18 h) significantly increased NO production in MC from SD rats, whereas IFN $\alpha / \beta$ but not IFN $\gamma$ increased $\mathrm{NO}$ production in $\mathrm{BN}$ MC. Agreater amount of NO was produced by SD MC compared to BN MC. Neither BN nor SD MC expressed iNOS mRNA constitutively, but iNOS mRNA expression was detected in SD MC after IFN $\gamma$ or IFN $\alpha / \beta$ stimulation. We have been unable to detect iNOS mRNA in BN MC, regardless of stimulation. To analyze the importance of availability of substrate and cofactors in NO production we added excess $\mathrm{L}$-arginine, or enhanced levels of L-sepiapterin, a precursor of the tetrahydrobiopterin cofactor. L-arginine did not increase $\mathrm{NO}$ production in $\mathrm{BN}$ or SD MC, whereas preincubation with sepiapterin potentiated IFN induced NO in both SD and BN MC. Thus, there are strain associated differences in NO production by MC that may relate to NOS expression and availability of some cofactors. Future studies will more fully characterize mechanisms underlying strain-associated differences in NO production and the potential relevance of such differences. Supported by CIHR.

\section{Identification of Noninvasive Markers of Asthma Airway Inflammation Using 1H-NMR Spectroscopy of Guinea Pig Urine}

Obiefuna, I.P. and Adamko, D.J., Pulmonary Research Group, University of Alberta, Edmonton, $A B$

Asthma is the most prevalent chronic illness of children, causing airway obstruction secondary to inflammation. Lung function testing would be helpful in managing treatment but this can be difficult or impossible in young children. To deal with this problem, we are using Nuclear Magnetic Resonance (NMR) spectroscopy, a non invasive metabonomic tool to identify markers of asthma inflammation in the urine. Metabonomics is the quantitative measurement of the dynamic multiparametric metabolic response of living systems to pathophysiological stimuli or genetic modification. We hypothesize that the metabolic activity of airway inflammatory cells associated with asthma will produce a unique pattern of proteins, which can be measured in the urine. Our preliminary data in humans suggests that NMR can detect asthma metabolites in the urine. To clearly establish the link between lung inflammation and urine markers, we are using a guinea pig model of asthma to correlate airway changes with urine markers.

Three groups of female guinea pigs were used: unsensitized control, sensitized alone $(10 \mathrm{mg} / \mathrm{ml}$ ovalbumin for 3 alternate days) and sensitized challenged (sensitized + $0.5 \%$ ovalbumin aerosol, 2 mins). Airway hyperreactivity was measured using penH. Animals were killed by an overdose of anaesthesia, bronchoalveolar lavage obtained for differential cell count, lungs for histology and bladder urine collected for $1 \mathrm{H}$ NMR analysis. So far we have been able to identify substances in the urine of the various guinea pig groups that are present in different concentrations. If NMR can measure consistent patterns of 
metabolic change in the three groups of animals, we believe that this will confirm urine NMR's ability to correlate lung disease with urine metabolites.

\section{Cyclin-Dependent Kinase 5 Regulates Granule Exocytosis but Not Superoxide Release in Human Peripheral Blood Eosinophils}

Solomon Olawole Odemuyiwa, Michael R. Logan, Francis Davoine, Melinda Benn, Chad Wu, Darryl J. Adamko, Redwan Moqbel, Pulmonary Research Group, University of Alberta, Edmonton, $A B$

Introduction: Eosinophils release stored granule mediators and reactive oxygen species and thus contribute to the pathogenesis of allergic asthma. However, the intracellular mechanisms governing the release of these mediators are poorly understood. Recent studies have suggested that cyclin-dependent kinase 5 (cdk5) may be important in the process of granule exocytosis in neurons, insulin-producing cells and neutrophils. Objectives: To determine the expression of cdk5, and cdk5 activators (p35 and p39), and their role in eosinophil activation and mediator release. Methods: Western blotting, RT-PCR and flow cytometry were used to determine the expression of cdk5, p35 and p39 in eosinophils obtained from atopic human donors. Following treatment with a specific inhibitor of cdk5, roscovitine, the release of eosinophil peroxidase (EPO) was measured in cells activated with IgA-coated beads or PMA. In addition, superoxide-dismutase-inhibitable superoxide production was measured in PMA- and secretory IgA-activated eosinophils by ferricytochrome c reduction. Intracellular calcium flux was also determined in fura-2-loaded eosinophils following treatment with roscovitine. Results: Specific primer pairs and antibodies detected mRNA and protein for cdk5 and p39 but not p35. Flow cytometry demonstrated that $18 \%$ of resting eosinophils expressed activated cdk5 compared to $45 \%$ expression following activation with IL-5. Treatment with $20 \mathrm{uM}$ roscovitine resulted in $60 \%$ inhibition of EPO release in IgA-bead-activated eosinophils. There was no significant difference in superoxide release in roscovitine-treated eosinophils compared to untreated cells. Granule mobilization, determined through surface expression of CD63 on activated eosinophils, was inhibited following treatment with roscovitine. Similarly, pre-treatment with roscovitine inhibited intracellular calcium flux following activation of eosinophils with PMA. Conclusions: cdk5 regulates eosinophil activation and mediator release. Local delivery of cdk5 inhibitors may reduce the inflammatory and physiological changes associated with allergic asthma in the respiratory airway. Funding: Canadian Institutes of Health Research, Alberta Heritage Foundation for Medical Research (AHFMR).

\section{Recombinant Fel d 1 Elicits a Different Pattern of Human Cytokine and Chemokine Responses Than Do Clinical Cat Extracts}

P. Pochard, Y. Lissitsyn, A. Silaghi, W.R. Thomas, S. Jones, A.L. Kozyrskyj, A.B. Becker, K.T. HayGlass, Department of Immunology, University of Manitoba, BMSB, Winnipeg, MB; National Microbiology Laboratory, Population and Public Health Branch, Canadian Science Centre for Human and Animal Health, Winnipeg, MB; TVW Telethon Institute for Child Health Research, West Perth, Australia; Department of Community Health Sciences, University of Manitoba; John Buhler Research Center, University of Manitoba

Introduction: Whole cat antigen extracts are commonly used for skin prick tests and for in vitro experimental analysis of immune capability. However, commercial preparations are unlikely to be free of contaminants, such as bacterial, viral and fungal components, that may influence the results obtained in analyses of immune status. Objective: Compare the in vitro immune response profile obtained with two commercial cat antigen extracts (ALK and Omega) that are widely used for skin prick tests to purified recombinant Fel d 1. Method: The presence, nature and quantity of TLR ligand contamination (ie, LPS) in commercial and recombinant cat Ag preparations was assessed using TLR-transfectants and LAL assays. These sources of cat Ag were used to stimulate peripheral blood mononuclear cells from over 60 clinically well-defined asthmatic and nonatopic children, born in 1995, in short-term primary culture. PBMC were Ag-stimulated for 24 hours or 5 days, time points found in preliminary experiments to yield optimal responses. Culture supernatants were assayed for allergen-driven cytokine and chemokine production by ultrasensitive ELISAs. Results: Acomparison between natural cat antigen extracts and $\mathrm{rFel} \mathrm{d} 1$ demonstrates that different profiles of cytokine/chemokine production are obtained with different sources, and purity, of Ag. At 24 hours, a timepoint reflecting the innate immune response, only the two commercial extracts induced IL-6 and IL-10 production associating strongly with the presence of TLR-ligand contaminants. Analysis of the Ag-specific recall response at day 5 revealed that among cat allergic children, rFel d 1 induced readily quantified $\mathrm{CD}^{4} \mathrm{~T}$ cell dependent production of cytokines (IL-13, low IL-10) and chemokines (CCL17, CCL22). Commercial cat antigen extracts elicit a clearly distinct pattern of cytokine production, characterized by a high level of IL-10, moderate IFN $\gamma$, and lower IL-13 responses. Type 2 chemokine production was also lower with these extracts compared to rFel $\mathrm{d} 1$. Conclusion: Our results clearly demonstrate the importance of the purity of the antigen used for immune 
analysis. They suggest the need to carefully assess existing data obtained with heterogeneous contaminated, Ag preparations in clinical and research settings.

\section{IL-17 Induces Production of IL-8 in Human Airway Smooth Muscle Cells via p38 MAPK, p42/p44 ERK, AP-1 and NF- $\kappa$ B Dependent Pathways}

Muhammad Shahidur Rahman, Andrew J. Halayko, Abdelilah Soussi Gounni, Department of Immunology, Physiology and Sections of Respiratory Diseases, University of Manitoba, Winnipeg, $M B$

Introduction: Both neutrophils and interleukin (IL)-8, a potent neutrophil attractant, have been shown to play a central role in the pathophysiology of acute lung disorders. IL-17 is implicated in the regulation of inflammation by inducing neutrophil recruitment and is found in increased amounts in the asthmatic airway. We have revealed that IL-17-stimulated human airway smooth muscle (ASM) cells are capable to release IL-8. In this study we have investigated the signaling pathways by which IL-17 regulates IL-8 production in ASM cells. Methods: ELISA and quantitative real-time PCR were carried out to investigate the effect of IL-17 on IL-8 protein and mRNA expression. Activation of $\mathrm{p} 38$ MAPK, p42/p44 ERK and STAT-6 in IL-17-stimulated ASM cells was clearly demonstrated by Western blotting. SB 203580, a specific inhibitor of p38 MAPK, U 0126, a specific inhibitor of $\mathrm{p} 42 / \mathrm{p} 44$ ERK and wortmannin, a inhibitor of PI-3K were used to investigate their involvement in IL-17-induced IL-8 release. Further, the involvement of AP-1 and NF- $\kappa \mathrm{B}$ was also assessed by transient transfection study. Results: IL-17 induced IL-8 protein release and mRNA expression from ASM cells. Using Western blot, we observed an activation of p38 MAPK and p42/p44 ERK, but not STAT-6 in IL-17 stimulated ASM cells. We also observed a significant decrease of IL- 8 release in the presence of p38 MAPK and $\mathrm{p} 42 / \mathrm{p} 44$ ERK inhibitors. Finally, IL-17 induced IL-8 release was mediated by $\mathrm{AP}-1$ and NF- $\kappa \mathrm{B}$ signaling pathways. Conclusion: Together these results provide grounds for targeting IL-17-associated pathways in therapeutic modulation of airway inflammation.

\section{Barriers for Families in Managing Their Child's Asthma}

N.L. Ross, C.A. Gillespie, B. Kulbaba, S.E. Filuk, L.J. Stewart, D.L. Stockwell, W.T.A. Watson, F.E.R. Simons, A.B. Becker, Children's Asthma Education Centre, University of Manitoba, Winnipeg, $M B$

In working with families to achieve good asthma control, The Children's Asthma Education Centre seeks to identify the barriers that make it difficult for families to manage their child's asthma. From January 2004 to May 2005 , we evaluated a group of 134 families who had committed to asthma education. Prior to their participation in the Family Asthma Program ${ }^{\odot}$ the families filled out a self-administered questionnaire. These families were asked if anything made it hard for them to care for their child's asthma. A checklist of possible barriers was presented to the families. For the 134 families, $68.6 \%$ checked off at least one barrier. Of the group of 134 the following specific barriers were identified.

- Lack of information about asthma, $29.9 \%$

- Concerns about using medicine, $27.6 \%$

- Hard to avoid things that cause problems, $25.4 \%$

- Different information from different people, $18.3 \%$

- Lack of written instructions for care, $15.7 \%$

- Hard to see the same doctor, $6.7 \%$

- Lack of money, $5.2 \%$

- Problems using the inhaler, 5.2\%

- Lack of time, $3.7 \%$

- Unable to understand instructions, 3.0\%

In addition families responded to open ended questions about the possible barriers created by other people and other reasons. The most common barrier identified by families was lack of information. Other common barriers are concern about using medicine and difficulty avoiding triggers that cause problems. Consideration of these factors may help asthma educators to develop effective strategies to help families achieve the goal of good asthma management.

\section{Impact of IgE Binding to Neutrophils in Asthma}

Arash Shoja Saffar, Lianyu Shan, Abdelilah Soussi Gounni, Department of Immunology, Faculty of Medicine, University of Manitoba, Winnipeg, $M B$

Introduction: Asthma is a chronic inflammatory disease with an increasing prevalence in developed countries. Although neutrophil activation has been demonstrated in various forms of asthma, a clear mechanism has not been identified yet. The high affinity IgE receptor ( $\mathrm{Fc} \notin \mathrm{RI}$ ), a classic molecular link in allergy, has recently been characterized by our team in neutrophils of atopic asthmatics. Methods: Neutrophils were isolated from peripheral blood of atopic asthmatics and incubated with IgE, GM-CSF or medium only for 18 hours. Apoptosis was assessed by annexin $\mathrm{V}$ binding and propidium iodide. Results: In this study, we found monomeric human IgE to protect neutrophils of atopic asthmatics from spontaneous apoptosis by binding to FceRI. This effect was not dependent on cross-linking of the receptor, autocrine release of mediators, or Fas/Fas ligand expression. Prevention of apoptosis seemed to occur through increased expression of the anti-apoptotic molecule Mcl-1, and stabilization of the pro-apoptotic molecule Bax in the cytoplasm. Furthermore, caspase activity and release of Smac from mitochondria were also partially down-regulated by binding of IgE to these 
cells. Conclusion: Taken together, the above results suggest that IgE in allergic asthma may delay programmed cell death of neutrophils and might therefore play a role in neutrophilic inflammation of airways.

\section{Nitration-a Possible Mechanism in Post- translational Control of Mast Cell Phenotype and Function}

Yokananth Sekar, Dean Befus, Pulmonary Research Group, Department of Medicine, University of Alberta, Edmonton, $A B$

Because mast cells (MC) are primary effector cells in allergic reactions, more knowledge about mechanisms that control their activities is essential. Both mast cell derived and exogenous nitric oxide (NO) down regulate many functions of MC. Nitration and nitrosylation are important mechanisms by which NO mediates posttranslational control of protein functions. We hypothesized that there are several molecular targets of NO-mediated post-translational control in $\mathrm{MC}$, and have begun to identify some of these nitrated targets. Western blot analysis of nitrated proteins from HMC-1 was assessed using rabbit polyclonal anti-nitrotyrosine antibody and rabbit IgG control antibodies. The $\mathrm{Mr}$ of the specific nitrated bands of untreated HMC-1 were estimated and compared with bands identified following treatment of HMC-1 cells with the NO donor SNOG (S-nitrosoglutathione), at different concentrations (100 to $1,000 \mathrm{uM}$ ) and at different time intervals (1 to $60 \mathrm{~min}$ ). Out of the nine bands that were identified in the unstimulated HMC-1 lysate, four bands of Mr 66, 54, 50 and $44 \mathrm{kDa}$ were $100 \%$ reproducible in four gels, whereas bands of $59,58,53,45$ and $38 \mathrm{kDa}$ appeared in three of four gels. Surprisingly, the number and intensity of nitrated proteins did not change in a stimulus-driven manner. We are currently using high-resolution two dimensional electrophoresis to improve the resolution of our studies. Protein identification by sequencing will help to focus on selected proteins and/or a particular signaling pathway that underlies the effects of NO in controlling MC phenotype and function. Supported by: Canadian Institutes of Health Research.

\section{Barriers to Establishing Local Asthma Education Resources in Rural Manitoba}

L.J. Stewart, C.A. Gillespie, N.L. Ross, S.E. Filuk, B. Kulbaba, F.E.R. Simons, W.T.A. Watson, A.B. Becker, Children's Asthma Education Centre, University of Manitoba, Winnipeg, $M B$

The Children's Asthma Education Centre (CAEC) has established a successful asthma education program for families in Winnipeg. In 2004 a project was initiated to assist other Manitoba communities to develop programs in their region. The CAEC Provincial Outreach Asthma
Coordinator contacted key players in Regional Health Authorities (RHA) outside of Winnipeg to identify local asthma education initiatives, priorities and goals, barriers to accomplishing goals, strategies to overcome barriers and resources available. Contact has also been made with Manitoba Health and the Health Programs and Services Executive Network (HPSEN) to discuss strategies to move asthma education forward in the province.

Through a series of interviews and a workshop organized by HPSEN, barriers to the establishment of local asthma education were identified as follows: (1) lack of awareness of the prevalence and impact of asthma in the community, (2) perceived lack of demand for education, (3) lack of financial support to train certified asthma educators and to support educational programs, (4) lack of human resources to deliver asthma education, (5) lack of a provincial strategy including coordination and standardization of asthma education resources, (6) geography/transportation where the population is scattered, (7) inconsistent care as a result of transient physician population, (8) limited professional awareness of current asthma guidelines and management, (9) lack of local champions, marketing or promotional strategies, (10) maturity of community readiness for behaviour change, (11) language and cultural concerns, eg: First Nations communities, (12) RHA changing priorities. In conclusion, it is critical to identify barriers to establishing local asthma education programs. Partnering with RHAs and local champions will be necessary to help identify strategies to overcome some of these barriers.

\section{Histone Deacetylation: Does It Play a Role in Recall Immunological Responses in Asthma/ Allergic Disease?}

R.-C. Su, A. Becker, A. Kozyrskyj, K.T. HayGlass, CIHR National Training Program in Allergy and Asthma Research, Departments of Immunology and C.H.S. Pediatrics/Child Health, University of Manitoba, Winnipeg, $M B$

Differential allergen-specific cytokine production and cytokine receptor expression are hallmarks of human allergic respiratory tract disease that are tightly integrated with the Th1/Th2 balance seen upon allergen reexposure in those exhibiting clinical tolerance vs. hypersensitivity. Very little is known about the molecular mechanisms through which the "memory" of the biased Th1/Th2 response is maintained upon allergen re-exposure. Cellular memory of recall immunological responses may be retained as epigenetic markings (i.e., covalent modifications of histones associated with DNA and DNA itself) on genes. Specifically, these markings include acetylation, methylation, phosphorylation and ubiquination of histone tails. Acetylation of histone tails 
is functionally associated with active gene expression. We hypothesize that inhibition of histone deacetylation (i.e., preventing gene silencing and enhancing gene expression) is associated with altered recall cytokine response in $\mathrm{PBMCs}$ from individuals with allergic disease.

To test this hypothesis, a short-term primary culture system using peripheral blood mononuclear cells (PBMC) was used. Fresh PBMC from 8 to 9 year old children were isolated and cultured with PHA (phytohemagglutinin) or HDM (house dust mite) in the presence or absence of a histone deacetylase inhibitor, TSA (trichostatin A). Supernatants were collected and the production of Th1 (IFN $\gamma$, IP-10), Th2 (IL-13, TARC), IL-1 $\beta$, and IL-10 was quantified by ELISA. Clinical parameters, such as physician-diagnosed asthma and rhinitis, in addition to skin prick test, and airway hyper-responsiveness measurements were used in grouping the subjects (allergic asthmatic versus non-atopic). Our preliminary results show that inhibition of histone deacetylase with TSA reduces Th1 responses and IL-10 production, and enhances Th2 responses during PHAstimulation. This is observed in both allergic and nonatopic PBMC cultures. Interestingly, inhibition of histone deacetylase with TSA reduces IL-13 production only in the non-atopic PBMC cultures and reduces IL$1 \beta$ production only in the allergic PBMC cultures. More cytokines and chemokines from a greater number of PBMC cultures will be analyzed to confirm these findings. This study will enhance our understanding of the induction and maintenance of recall responses. Understanding epigenetic regulatory mechanisms may provide new strategies to alter the type of recall responses. This study is supported by the National Training Program in Allergy and Asthma Research and CIHR Canada Research Chair in Immune Regulation.

\section{Involvement of Syk Tyrosine Kinase in Regulation of Pro-inflammatory Signaling in Lung Epithelium is Mediated by MAPK}

Marina Ulanova, Marcelo Marcet-Palacios, Samuel Asfaha, Alan D. Schreiber, A. Dean Befus, University of Alberta, Edmonton, AB; University of Pennsylvania School of Medicine, Philadelphia, PA

Syk kinase is best known as a critical component of immunoreceptor signaling in leukocytes. We have recently found that Syk is widely expressed in lung epithelial cells (LEC) and participates in $\beta 1$ integrin signaling. In the present study we assessed downstream signaling pathways regulated by Syk in LEC. Human bronchial epithelial cell line HS-24 was stimulated by TNF in vitro that caused the increase in expression of pro-inflammatory molecules IL-6 and ICAM-1, as well as of inducible nitric oxide synthase (iNOS) and, correspondently, production of NO. Inhibition of Syk using siRNA or piceatannol caused down-regulation of the expression of IL-6, ICAM-1, iNOS, and of NO production. Interestingly, Syk inhibition affected the expression of pro-inflammatory molecules only when LEC were simultaneously stimulated via $\beta 1$ integrins, suggesting that these receptors provide co-stimulatory signals up-regulating the pro-inflammatory effects of TNF. Syk involvement in regulation of LEC activation by TNF was mediated by the MAPK cascade. Indeed, inhibition of Syk using siRNA or piceatannol caused down-regulation of TNF-induced p38 and p44/42 MAPK phosphorylation, as well as reduced nuclear translocation of $\mathrm{p} 65$ NF- $\kappa$ B. Thus, TNF-induced activation of proinflammatory signaling in LEC is dependent on Syk and involves signaling pathways initiated by integrin receptor engagement. Further, the data suggest that Sykmediated signaling regulates the expression of proinflammatory molecules at least partly via activating the MAPK cascade. Understanding the role of Syk in signaling mechanisms in LEC may help in developing new therapeutic tools for inflammatory disorders such as asthma, COPD and acute lung injury. Funded by CIHR, CSACI/ CAAIF/Merck Frosst, Alberta Heritage Foundation for Medical Research and NIH.

Published: 15 September 2005

doi:10.1186/1710-1492-1-3-101

Cite this article as: Eck et al:: Annual Scientific Meeting, Winnipeg,

September 22-25, 2005. Allergy, Asthma \& Clinical Immunology 2005 1:101.

Submit your next manuscript to BioMed Central and take full advantage of:

- Convenient online submission

- Thorough peer review

- No space constraints or color figure charges

- Immediate publication on acceptance

- Inclusion in PubMed, CAS, Scopus and Google Scholar

- Research which is freely available for redistribution

Submit your manuscript at www.biomedcentral.com/submit
C Biomed Central 